\title{
Essais effectués en U.R.S.S. sur des turbines hydrauliques en service
}

\author{
Tests carried out in the U.S.S.R. \\ on hydraulic turbines in service
}

\author{
PAR N.N. KOVALEV \\ PRofesseuh a L'institut des Études de Machines de L'ACadímie des Sciences de L'U.r.s.s.
}

(Notes et documents présentés hors programme lors des Vos Journées de l'Hydraulique)

\begin{abstract}
Afin de mieux connaitre les conditions de travail des grosses turbines hydrauliques, l'usine L.M.Z. de Leningrad (U.R.S.S.) s'est livrée à des mesures concernant les pressions, les déformations et les vibrations intervenant sur les pales des turbines en fonctionnement. Description sommaire de l'appareillage utilisé et de certains résultats obtenus sur l'une des 20 turbines type Kaplan, de $126.000 \mathrm{kVA}$, de la centrale de Konibychev.
\end{abstract}

En vue de la production, de grosses turbines hydrauliques à grande puissance, l'usine métallurgique de Leningrad (L.M.Z.) a effectué des essais de déformation, de pression et de vibration sur les pales de turbines hydrauliques. Des essais de cette nature ont été effectués également dans plusieurs centrales hydroélectriques et en particulier sur l'une des vingt turbines (dont on peut voir la coupe sur la figure 1) équipant la centrale de Kouibychev. C'est aussi au cours de la préparation des pièces à l'usine métallurgique de Leningrad qu'ont été faits les travaux en prévision de l'installation des capteurs et des câbles, ainsi que des dispositifs de mesure.

La figure 2 représente la disposition des appareils de mesure. Les conducteurs passent par des dispositifs hermétiques à partir de capteurs fixés sur les pales et entrent dans le moyeu de la roue, où on a prévu une longueur libre de câble en vue de compenser son allongement pendant la fermeture des pales. Dans la partie inférieure du moyeu est placée une boîte hermétique renfermant un dispositif de commutateurs ainsi que d'autres éléments d'appareillage. De cette boîte hermétique, les conducteurs vont, dans des tubes à câbles spéciaux sis à l'intérieur de l'arbre, vers

\begin{abstract}
In order to gain a fuller lnowledge of the operating conditions of large hydraulic turbines, the L.M.Z. factory in Leningrad (U.S.S.R.) have carried out measurements of pressure, deformation and vibration occuring in turbine blades during operation. Brief description of the instrumentation used, together with some results obtained on one of the twenty 126,000 kVA Kaplan type turbines installed in the Kuibychev power station.
\end{abstract}

le distributeur d'huile placé au-dessus du générateur de courant. Sur le distributeur d'huile, on a installé une prise de courant (fig. 3) à partir de laquelle les conducteurs sont reliés à un appareillage (fig. 4 et 5) placé dans la salle des machines. Les appareils électroniques enregistreurs ont été préparés par l'Institut des Etudes de machines de l'Académie des Sciences de l'U.R.S.S. Sur l'une des six pales de la turbine, ont été installés 60 capteurs de pression (fig. 6) et 10 capteurs de vibrations; sur une autre pale, on a installé 80 capteurs de déformations; un certain nombre de capteurs de pression ont été placés sur la ceinture de la roue, et des capteurs de déformations sur l'arbre de la turbine, etc.

Nous avons éprouvé des difficultés en ce qui concerne la protection mécanique et électrique des capteur's contre l'action d'un courant d'eau tombant sur une pale avec une très grande vitesse.

Après un grand nombre d'essais effectués dans plusieurs centrales, nous avons essayé la turbine installée à Kouibychev, de la manière suivante : sur la surface d'une pale minutieusement nettoyée a été collé un capteur spécial, protégé par une grille spéciale faite de bandes d'acier soudées sur la pale. Ensuite, ceitc grille d'acier a été imprégnée de colle, puis séchée au 


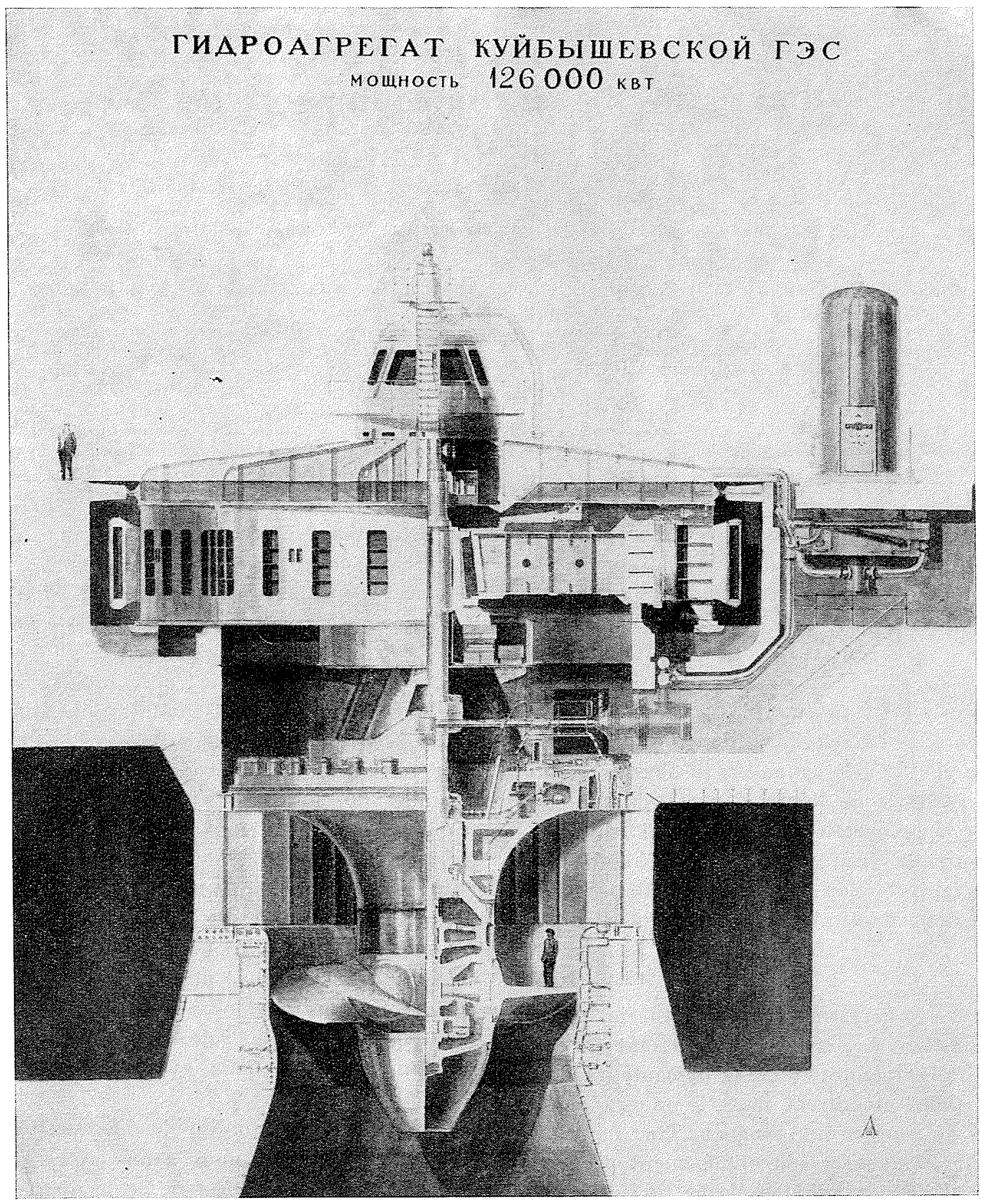

FIG. 1.

L'une des vingt turbines de $126.000 \mathrm{kVA}$ de la centrale de Kouibychev. 


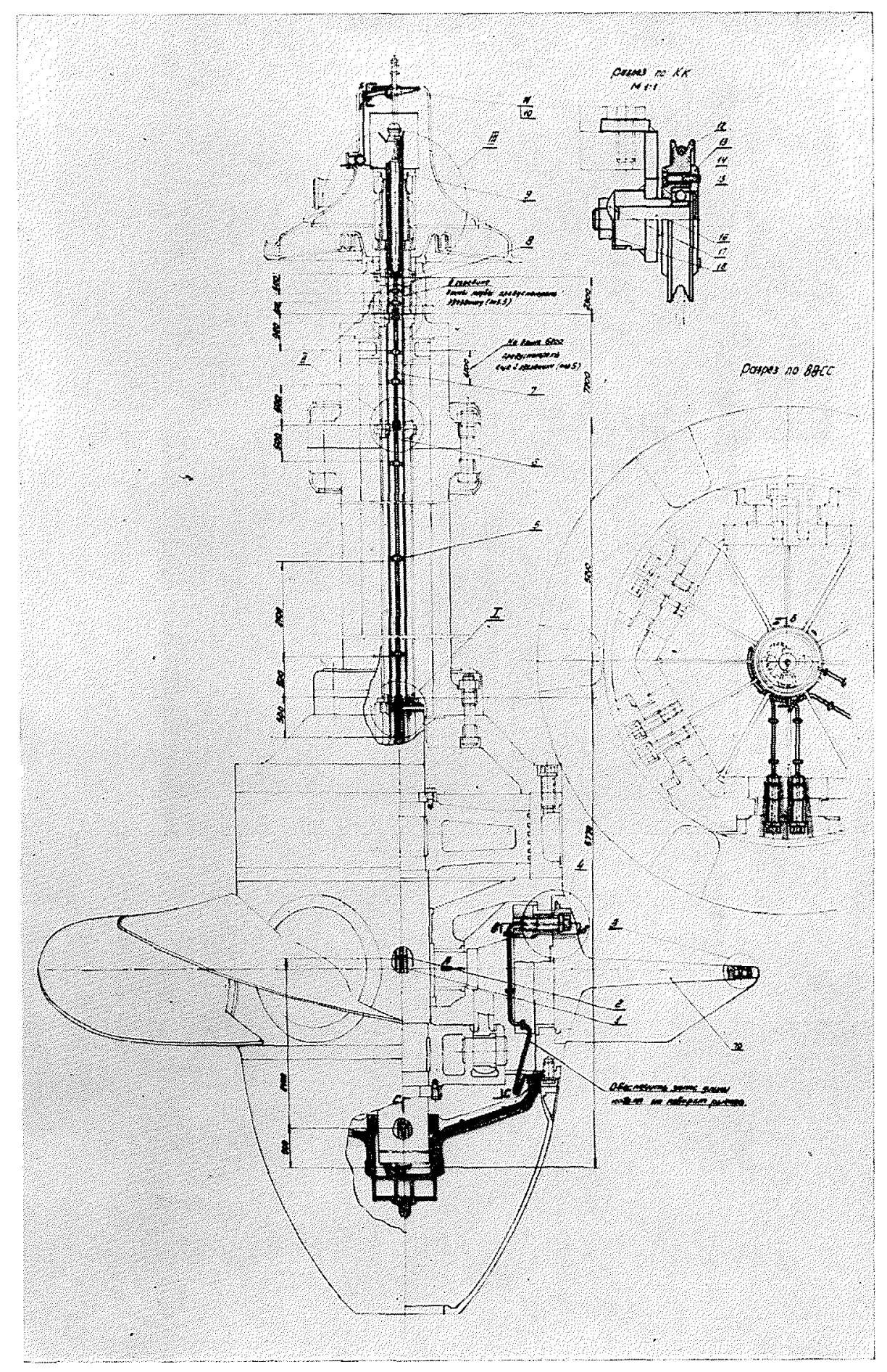

Fra. 2.

Disposition des appareils de mesure. 


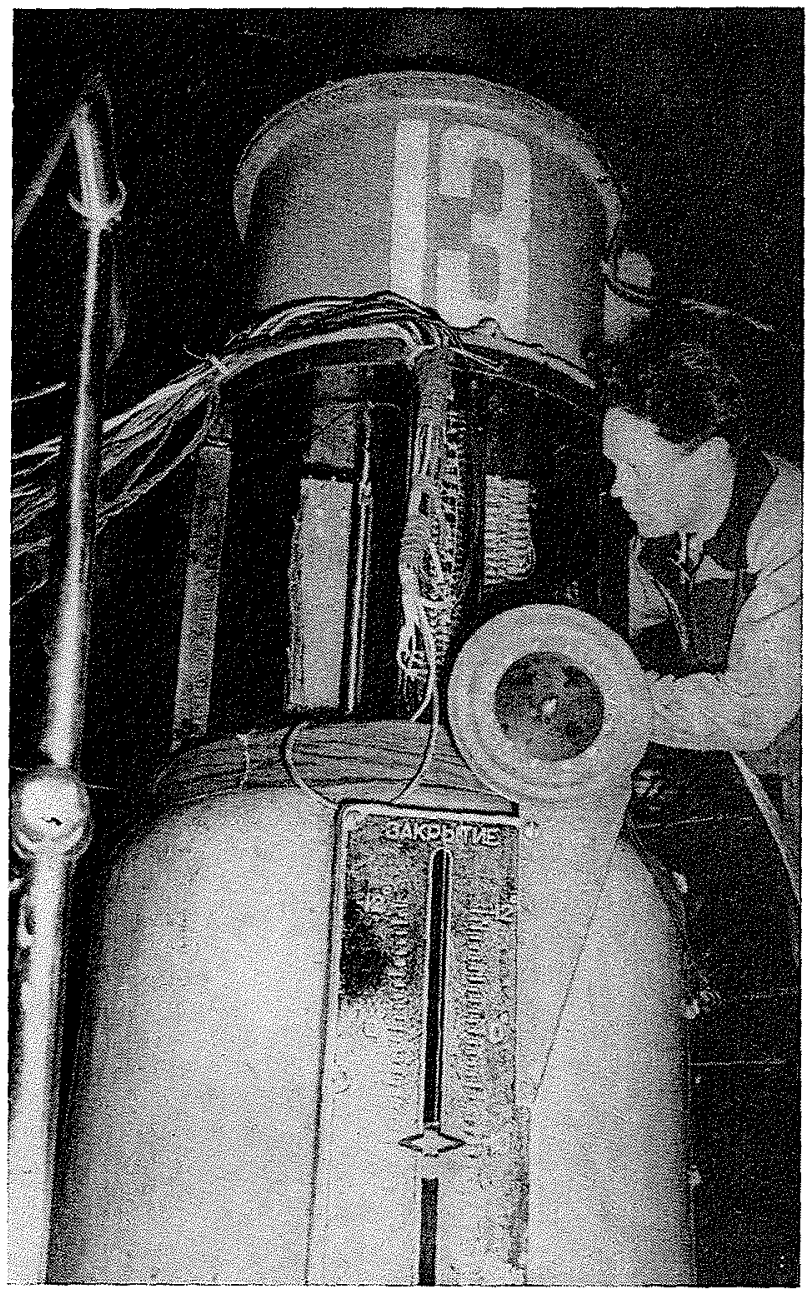

Fig. 3.

Prise de courant installée sur lẹ distributeur d'huile.

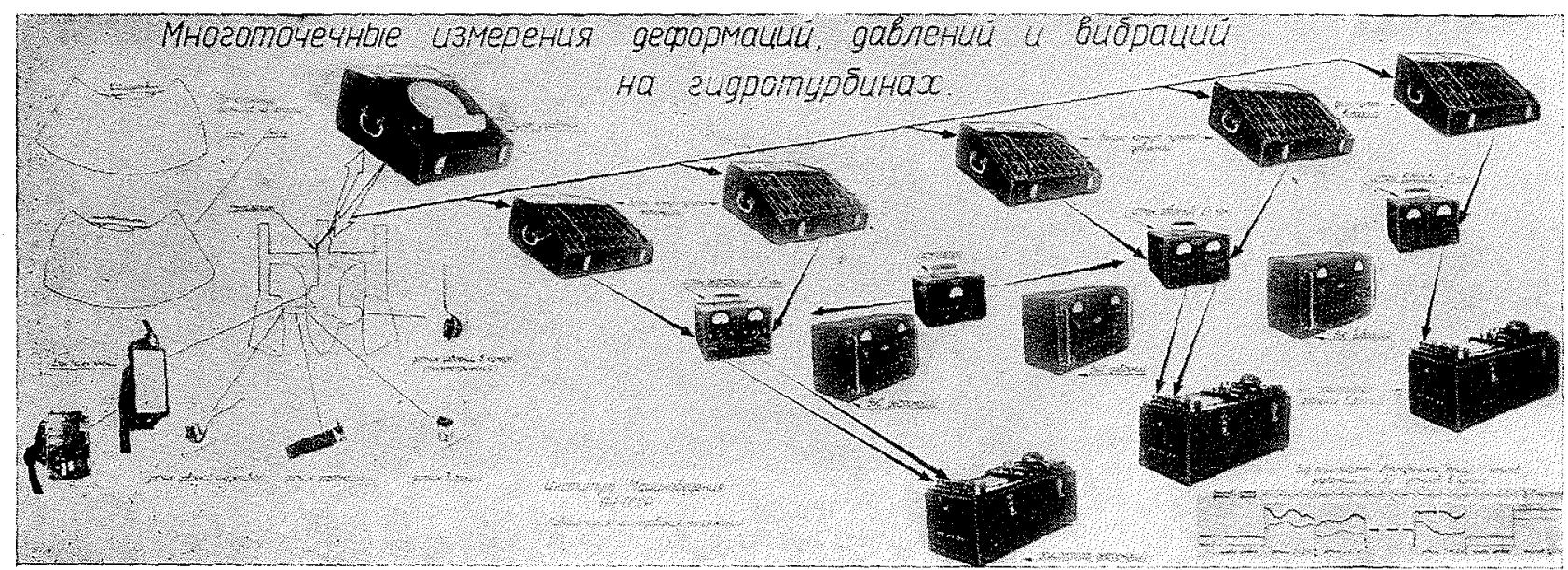

Fig. 4.

Appareillage de mesure. 


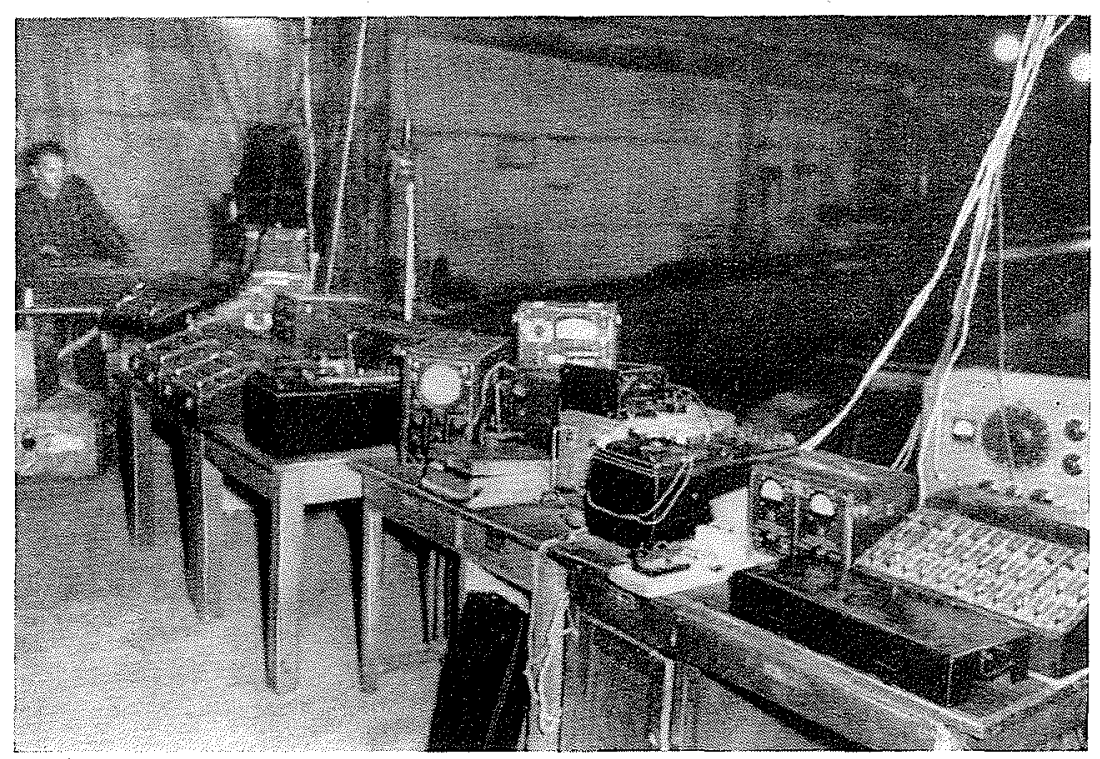

FIc. 5 ,

Appareillage de mesure.

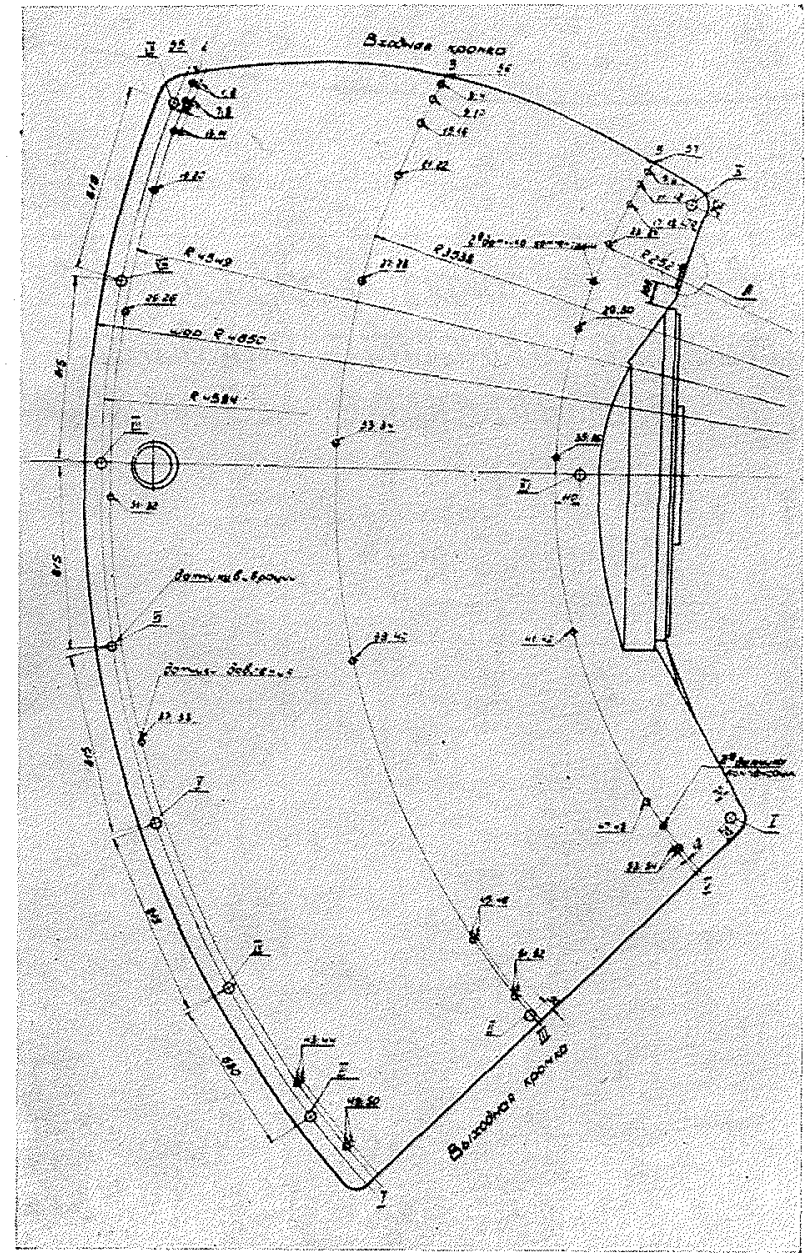

FIG. 6.

Une pale avee les 60 capteurs de pression. 


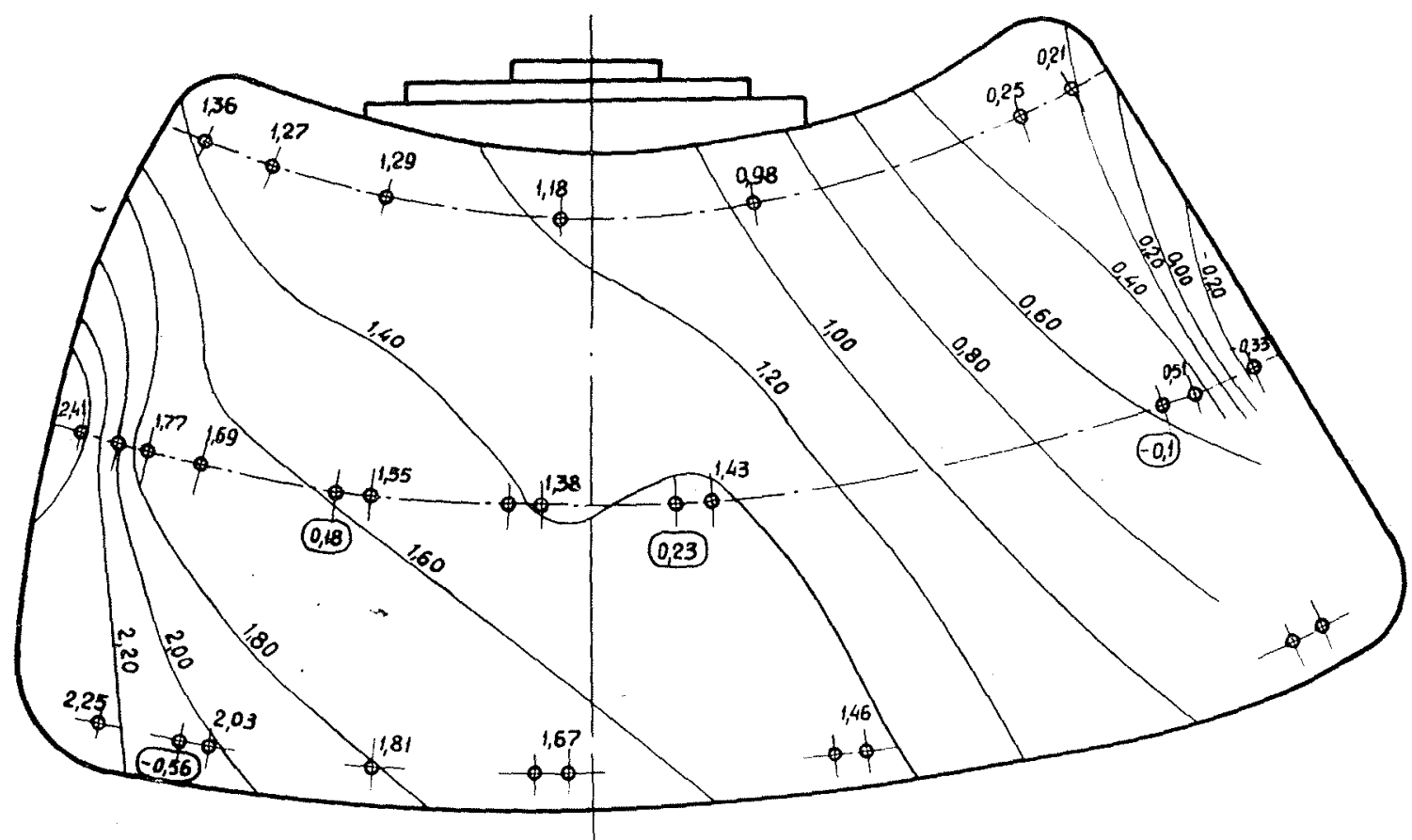

FIg. 7.

Lignes d'égale pression sur les pales en régime permanent. - N=30 MVA.

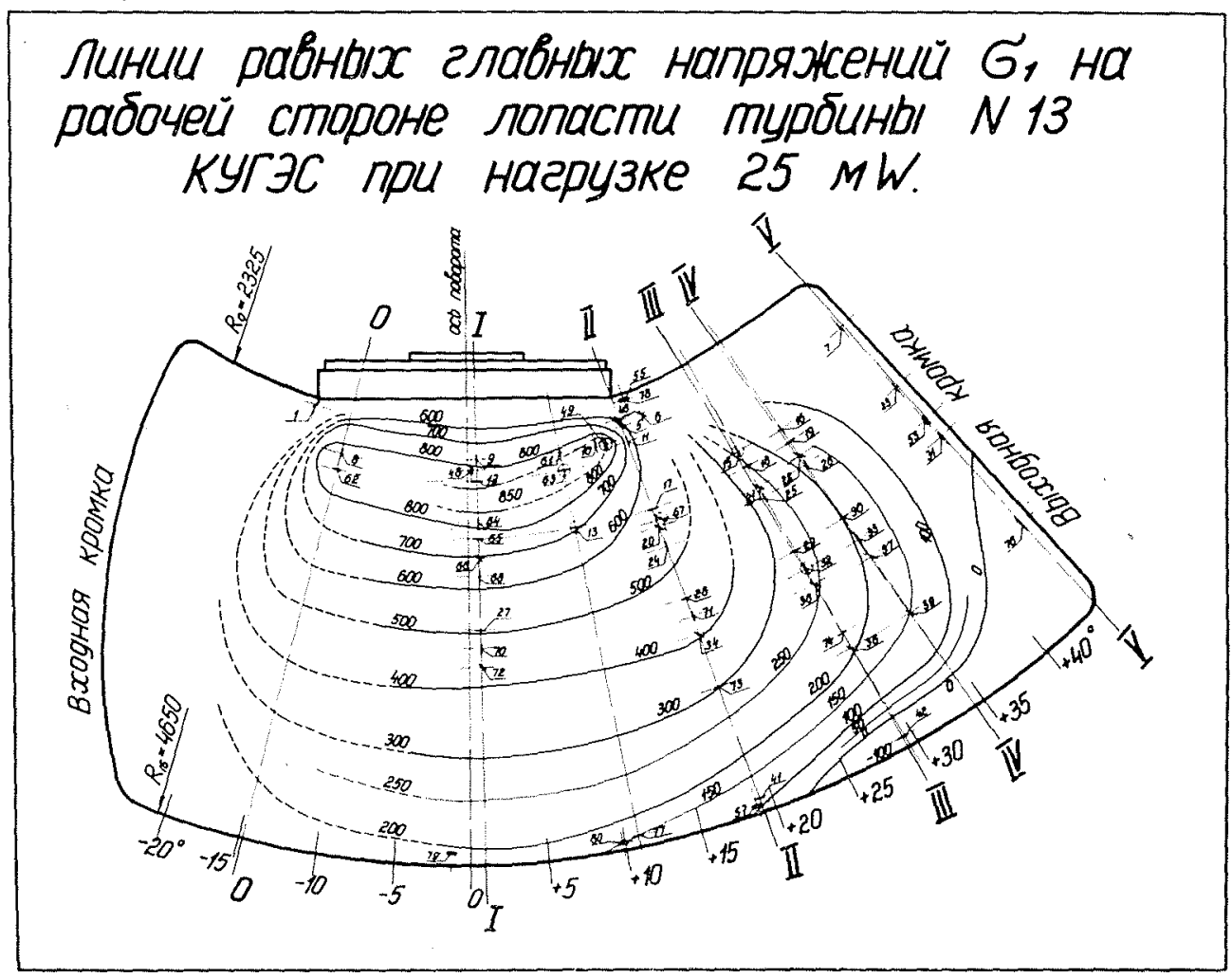

Fig. 8.

Lignes d'égales contraintes principales $\sigma$ sur l'intrados d'une pale de la turbine $\mathrm{N} 13$ de la centrale de Kouibychev, pourl une charge de 25 MTV. 
moyen d'échauffeur's spéciaux, ce qui a assuré une haute isolation ainsi qu'une assez grande robustesse mécanique du revêtement pendant la durée des essais.

Les essais de la turbine dans la centrale hydroélectrique de Kouibychev ont été effectués sous différentes charges normales et en régimes transitoires (démarrage, arrêt, décharge, survitesse).

Comme résultats de ces essais, nous avons obtenu les valeurs des tensions, déformations, pressions et vibrations des pales de la roue et d'autres pièces, pour des régimes variés de la turbine. Plusieurs milliers de valeurs mesurées ont été enregistrés sur des centaines d'oscillogrammes. Nous signalons à titre d'exemple la figure 7 où est représentée la pression de l'eau sur les pales en régime constant.

Les déformations et pulsations de la pression alternative maximum ont été observées dans un intervalle de 20 à $30 \%$ de la charge nominale. La figure 8 représente les courbes des contraintes dans les pales de la turbine. Sur la figure 9, on voit les variations de la pression, de la tension et du moment de rotation sur le tourillon des pales quand se produit une variation de vitesse pendant la survitesse et l'arrêt de la turbine.

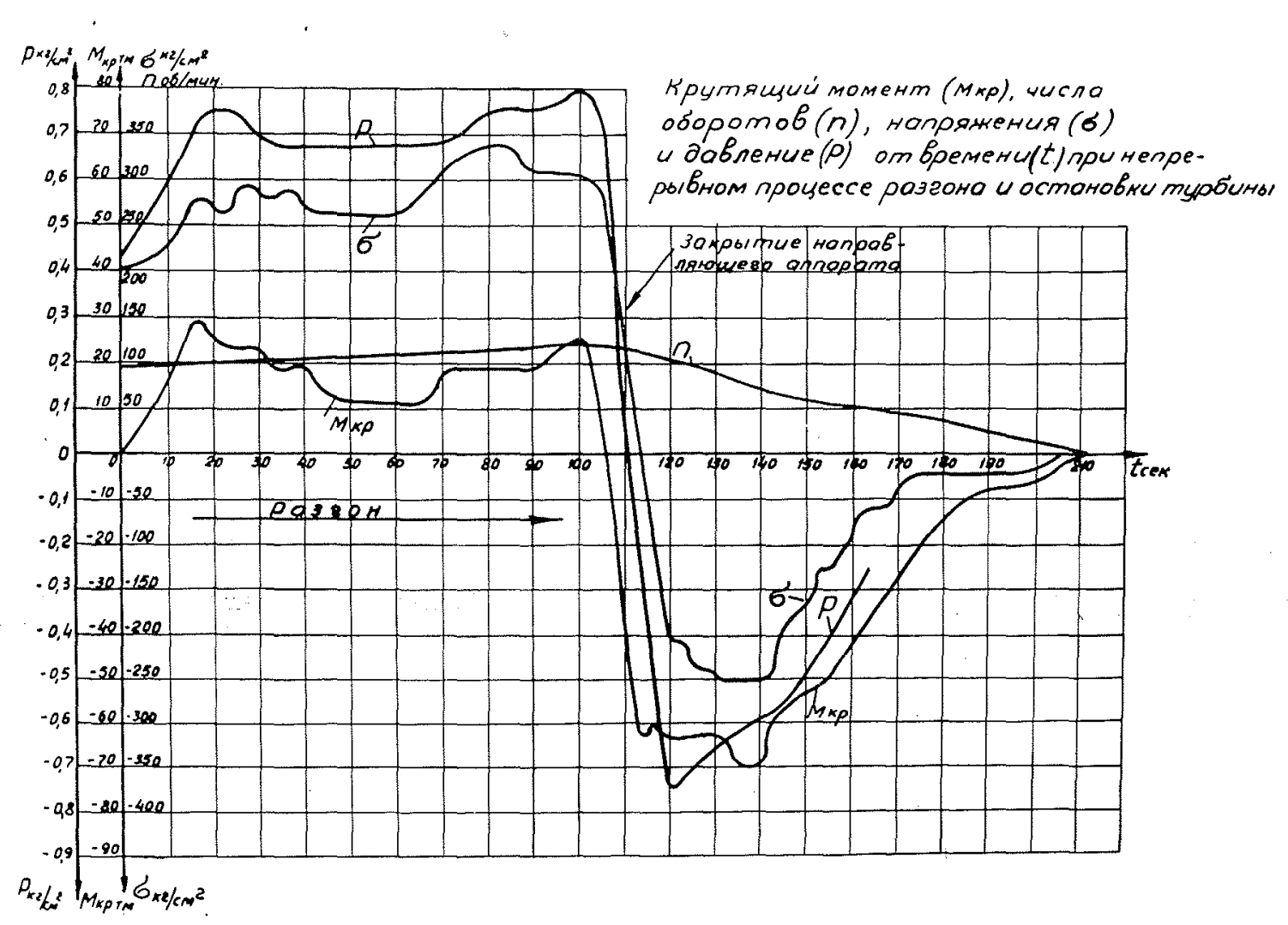

Fig. 9.

Couple de torsion (M), vitesse de rotation $(n)$, contrainte $(\sigma)$ et pression $(p)$ en fonction du temps $(t)$ lors d'une fermeture après disjonction et de l'arrêt de la turbine.

Partie supérieure de la figure: fermeture du distributeur.

Partie inferienre de la figure: emballement ( $t$ en secondes). 